\title{
Efficacy and outcomes of facial nerve-sparing treatment approach to cerebellopontine angle meningiomas
}

\author{
*Randy S. D’Amico, MD, ${ }^{1}$ Matei A. Banu, MD, ${ }^{1}$ Petros Petridis, MS, ${ }^{1}$ Alexandra S. Bercow, BA, ${ }^{1}$ \\ Hani Malone, MD, ${ }^{1}$ Moshe Praver, MD, ${ }^{1}$ Tony J. C. Wang, MD, ${ }^{2}$ Steven R. Isaacson, MD, ${ }^{1,2}$ and \\ Michael B. Sisti, MD ${ }^{1,2}$
}

Departments of ${ }^{1}$ Neurological Surgery and ${ }^{2}$ Radiation Oncology, Columbia University Medical Center, New York, New York

\begin{abstract}
OBJECTIVE Advanced microsurgical techniques contribute to reduced morbidity and improved surgical management of meningiomas arising within the cerebellopontine angle (CPA). However, the goal of surgery has evolved to preserve the quality of the patient's life, even if it means leaving residual tumor. Concurrently, Gamma Knife radiosurgery (GKRS) has become an acceptable and effective treatment modality for newly diagnosed, recurrent, or progressive meningiomas of the CPA. The authors review their institutional experience with CPA meningiomas treated with GKRS, surgery, or a combination of surgery and GKRS. They specifically focus on rates of facial nerve preservation and characterize specific anatomical features of tumor location with respect to the internal auditory canal (IAC).

METHODS Medical records of 76 patients with radiographic evidence or a postoperative diagnosis of CPA meningioma, treated by a single surgeon between 1992 and 2016, were retrospectively reviewed. Patients with CPA meningiomas smaller than $2.5 \mathrm{~cm}$ in greatest dimension were treated with GKRS, while patients with tumors $2.5 \mathrm{~cm}$ or larger underwent facial nerve-sparing microsurgical resection where appropriate. Various patient, clinical, and tumor data were gathered. Anatomical features of the tumor origin as seen on preoperative imaging confirmed by intraoperative investigation were evaluated for prognostic significance. Facial nerve preservation rates were evaluated.
\end{abstract}

RESULTS According to our treatment paradigm, $51(67.1 \%)$ patients underwent microsurgical resection and $25(32.9 \%)$ patients underwent GKRS. Gross-total resection (GTR) was achieved in 34 (66.7\%) patients, and subtotal resection (STR) in $17(33.3 \%)$ patients. Tumors recurred in $12(23.5 \%)$ patients initially treated surgically, requiring additional surgery and/or GKRS. Facial nerve function was unchanged or improved in 68 (89.5\%) patients. Worsening facial nerve function occurred in $8(10.5 \%)$ patients, all of whom had undergone microsurgical resection. Upfront treatment with GKRS for CPA meningiomas smaller than $2.5 \mathrm{~cm}$ was associated with preservation of facial nerve function in all patients over a median follow-up of 46 months, regardless of IAC invasion and tumor origin. Anatomical origin was associated with extent of resection but did not correlate with postoperative facial nerve function. Tumor size, extent of resection, and the presence of an arachnoid plane separating the tumor and the contents of the IAC were associated with postoperative facial nerve outcomes.

CONCLUSIONS CPA meningiomas remain challenging lesions to treat, given their proximity to critical neurovascular structures. GKRS is a safe and effective option for managing CPA meningiomas smaller than $2.5 \mathrm{~cm}$ without associated mass effect or acute neurological symptoms. Maximal safe resection with preservation of neurological function can be performed for tumors $2.5 \mathrm{~cm}$ or larger without significant risk of facial nerve dysfunction, and, when combined with GKRS for recurrence and/or progression, provides excellent disease control. Anatomical features of the tumor origin offer critical insights for optimizing facial nerve preservation in this cohort.

https://thejns.org/doi/abs/10.3171/2016.10.JNS161982

KEY WORDS cerebellopontine angle; meningioma; brain tumor; intracranial; skull base; Gamma Knife; microsurgery; oncology

ABBREVIATIONS CN = cranial nerve; $\mathrm{CPA}$ = cerebellopontine angle; EMG = electromyography; $\mathrm{EOR}$ = extent of resection; GKRS = Gamma Knife radiosurgery; GTR = gross-total resection; IAC = internal auditory canal; STR = subtotal resection; TTP = time to progression.

SUBMITTED August 3, 2016. ACCEPTED October 13, 2016.

INCLUDE WHEN CITING Published online February 10, 2017; DOI: 10.3171/2016.10.JNS161982.

* Drs. D'Amico and Banu contributed equally to this work. 
$\mathrm{T}$ UMORS of the cerebellopontine angle (CPA) account for approximately $10 \%$ of all intracranial tumors in adults. ${ }^{6}$ Meningiomas, the second most common tumor of the CPA after vestibular schwannomas, are slow-growing, benign lesions that account for 5\%-15\% of tumors in this region. Fortunately, meningiomas can often be cured by complete removal. As a result, major advances in microsurgical techniques, neuromonitoring, and image guidance have been made to improve surgical outcomes and reduce morbidity. ${ }^{2,21}$

Meningiomas arising within the CPA pose unique and significant surgical challenges, and, despite advances in microsurgical adjuncts and techniques, gross-total resection (GTR) cannot always be achieved without placing the patient at significant risk of morbidity and mortality. Damage to the facial nerve (cranial nerve [CN] VII) is a particularly adverse outcome of resection, and disfiguring and disabling facial paralysis has been demonstrated to significantly detract from patient quality of life. ${ }^{14,16}$ In general, the morbidity associated with CPA meningiomas is attributed to the way the tumors surround or infiltrate nearby cranial nerves and/or the brainstem..$^{5}$ In light of this, the goal of surgery has evolved to preserve the quality of the patient's life, even if it means leaving residual tumor. This is in part due to the concurrent rise of Gamma Knife radiosurgery (GKRS) as an acceptable treatment modality for newly diagnosed, recurrent, or progressive meningiomas within the posterior fossa, with large series demonstrating excellent tumor control rates and minimal adverse effects. ${ }^{2-4,8,12,20,25}$

In the present study, we review the experience of a single surgeon over the past 24 years in the treatment of meningiomas of the CPA treated with GKRS, surgery, or a combination. We use a treatment approach in which GKRS is the first treatment option for tumors smaller than $2.5 \mathrm{~cm}$, with the option of aggressive resection using facial nerve-sparing microsurgical techniques for tumors 2.5 $\mathrm{cm}$ or larger with adjuvant GKRS for recurrent disease or progressive residual. We have previously demonstrated the effectiveness of this approach for the treatment of vestibular schwannoma with preservation of good facial nerve function in $97 \%$ of treated patients. ${ }^{9}$ Here, we pay specific attention to rates of facial nerve preservation and investigate the clinical and prognostic significance of the tumor origin along the petrous bone with respect to the internal auditory canal (IAC).

\section{Methods}

\section{Patient Selection and Data Collection}

The Columbia University Institutional Review Board approved all aspects of this study. The medical records of 76 patients treated between 1992 and 2016 were retrospectively reviewed. Patients were included in the study if they had a previously undiagnosed lesion of the CPA, with both clinical and neuroimaging features consistent with a meningioma confirmed by 2 independent reviewers. The tumors were treated with either microsurgical resection performed by a single surgeon (M.B.S.) or GKRS performed by a team comprising the same surgeon and a radiation oncologist (S.R.I. or T.J.C.W.). Clinical features included a medical history absent of prior cancer and an intracranial tumor located in the posterior fossa with MRI and/or CT imaging features consistent with a meningioma. Neuroimaging features included extraaxial location, contrast enhancement, dural attachment, and calcification when apparent.

Meningiomas of the CPA were defined as tumors whose maximal volume was centered at the anatomical junction of the lateral cerebellum, the pons, and the internal auditory meatus. The bulk of the tumor was superior to the jugular foramen and inferior to the tentorial edge. We did not include meningiomas that arose primarily from the middle of the clivus or the foramen magnum. Tumor origin was classified as petroclival if it arose from the petroclival junction medial to the trigeminal nerve, displacing it laterally; anterior petrous if it arose along the petrous bone medial to the IAC and lateral to the trigeminal nerve, pushing it rostrally or medially; posterior petrous if it arose lateral to the IAC along the petrous bone; and tentorial if it arose from the tentorial edge and projected into the CPA, displacing the trigeminal nerve caudally. ${ }^{13}$ Particular attention was paid to the presence or not of an arachnoid plane between the tumor and the contents of the IAC intraoperatively.

Exclusion criteria included surgery for a presumed meningioma with a diagnosis of vestibular schwannoma or other nonmeningothelial tumor, or diagnosis of neurofibromatosis Type 2. Patients with multiple meningiomas in various intracranial locations, including a treated lesion within the CPA, were included, and data related only to the CPA lesion were collected. Histological diagnosis was confirmed in all patients treated with microsurgical resection. Various patient, clinical, and tumor data were gathered from the medical records, including demographics, pre- and postoperative neurological examinations, procedural details, tumor pathology, tumor size, and treatment characteristics. Postoperative MR images were evaluated when available to determine the extent of resection (EOR) and presence or absence of recurrence or residual.

\section{Treatment Paradigm}

Patients were selected for GKRS or a facial nervesparing microsurgical approach according to tumor size and symptoms, as depicted in Fig. 1. Patients with tumors smaller than $2.5 \mathrm{~cm}$ in the largest diameter and mild symptoms were treated with GKRS (see below). Patients with tumors $2.5 \mathrm{~cm}$ or larger in the largest diameter were treated with microsurgical resection. ${ }^{9}$ Exceptions were made at the discretion of the surgeon in the setting of significant comorbidities, advanced age, debilitating symptoms of vertigo or tinnitus, or significant mass effect on surrounding structures. These patients were analyzed within the group in which they were ultimately treated. Hearing preservation was not the primary goal of this treatment paradigm, but all efforts were made to preserve hearing when possible.

\section{Surgical Technique}

Surgery was performed in the lateral position via a ret- 


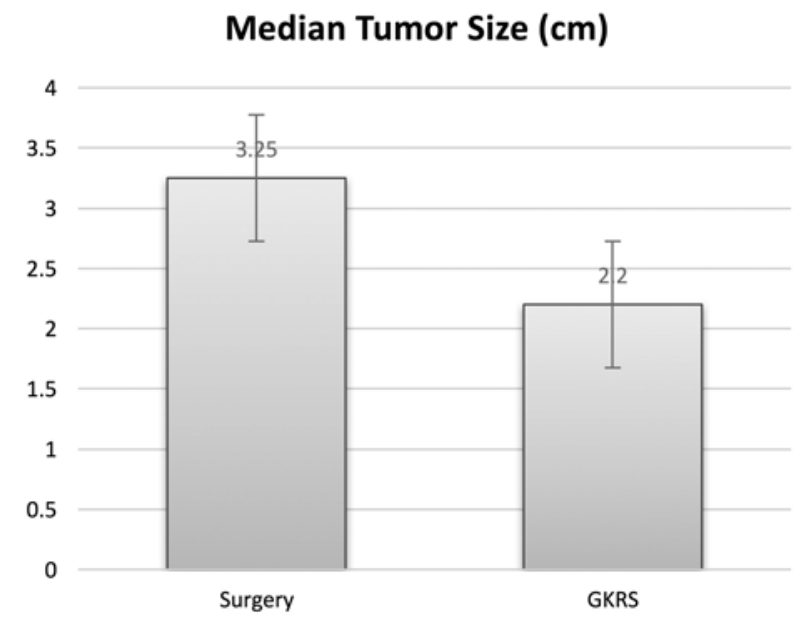

Median Tumor Size $(\mathrm{cm})$

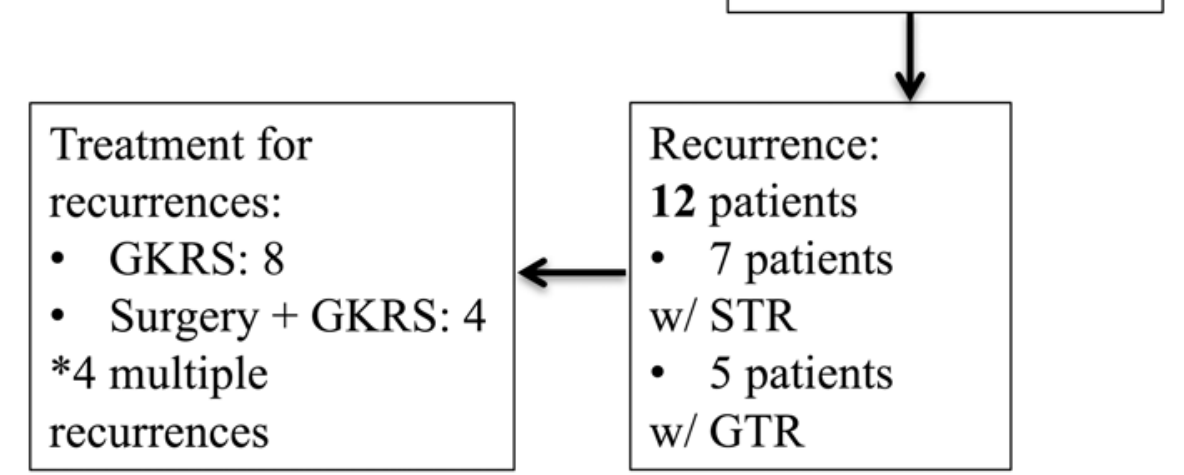

76 patients with CPA meningioma
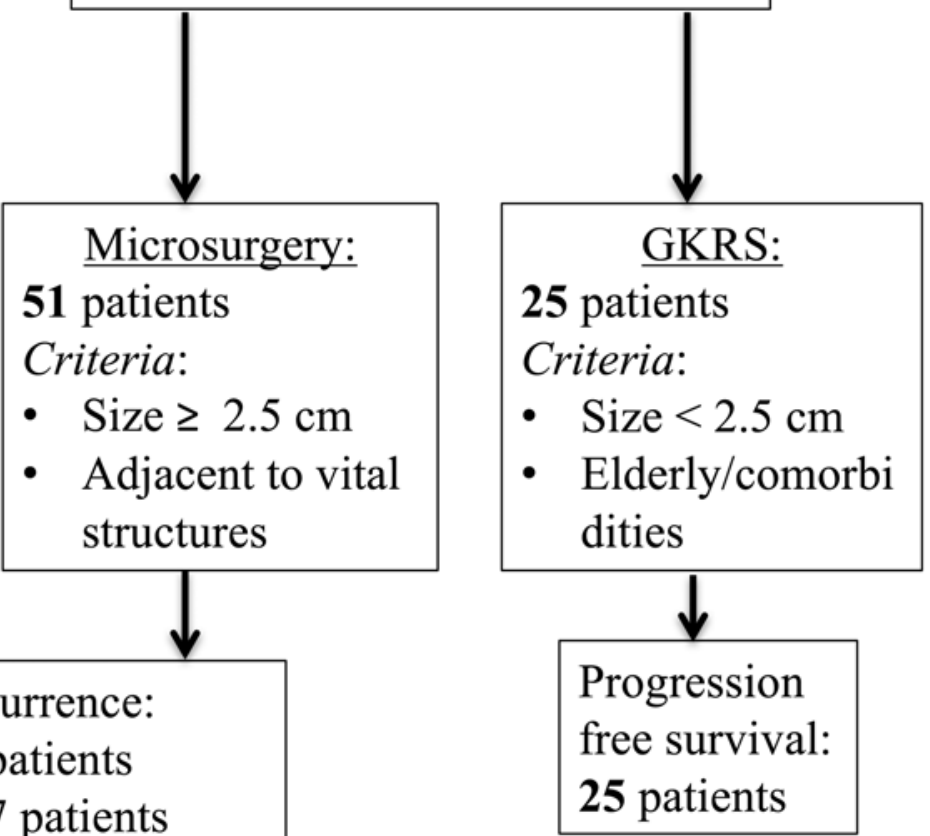

FIG. 1. Flow chart showing the treatment paradigm for CPA meningiomas and follow-up criteria. Patients with tumors smaller than $2.5 \mathrm{~cm}$ in the largest diameter and mild symptoms were treated with GKRS. Patients with tumors $2.5 \mathrm{~cm}$ or larger in the largest diameter were treated with microsurgical resection. The median tumor size was $3.25 \mathrm{~cm}$ (range 1.6-6.5 cm) and $2.2 \mathrm{~cm}$ (range $0.8-3.7 \mathrm{~cm})$ in patients treated initially with microsurgical resection and GKRS, respectively $(p<0.001)$. Exceptions were made at the discretion of the surgeon in the setting of significant comorbidities, advanced age, debilitating symptoms of vertigo or tinnitus, or significant mass effect on surrounding structures. Patients with histological diagnosis of a tumor other than meningioma, a known diagnosis of neurofibromatosis Type 2, or prior treatment of their tumor were excluded from the analysis.

rosigmoid approach (surgeon's preference). Electrophysiological monitoring and intraoperative image guidance were performed according to standard protocol at our institution. Gross-total resection (GTR) was attempted in all patients who underwent resection and was defined as the complete absence of the lesion on postoperative MRI. Subtotal resection (STR) was performed in the setting of any perceived threat to critical neurological and/or vascular structures. Intraoperative electrophysiological monitoring was performed in all cases according to standard protocol. This included brainstem auditory evoked responses, ipsilateral facial electromyography (EMG), and bilateral somatosensory evoked potentials. Evidence of potential facial nerve injury on intraoperative electrophysiological monitoring was defined by the requirement of greater than $0.3 \mathrm{~mA}$ stimulation, or greater than $0.1 \mathrm{~mA}$ stimulation above baseline on facial EMG. The porus acusticus was drilled as necessary to facilitate resection of tumors involving the IAC only if they were deemed low risk for potentially damaging the facial nerve based on intraoperative electrophysiological monitoring and deemed necessary for maximizing EOR per the attending surgeon. No patients underwent preoperative embolization.

\section{Radiosurgical Technique}

GKRS was performed for patients with tumors smaller than $2.5 \mathrm{~cm}$ and associated mild symptoms. In addition, GKRS was performed in the setting of progression of known residual disease after STR or recurrence in the setting of previous GTR. Briefly, GKRS began with application of the Leksell model G stereotactic frame (Elekta AB) after administration of a local anesthetic. After frame placement, high-resolution stereotactic MRI was performed. Thin-slice axial images were obtained after intravenous contrast administration. Radiosurgical dose planning was then performed by the neurosurgeon (M.B.S.) in conjunction with a radiation oncologist (S.R.I. or T.J.C.W.) and a medical physicist. The median prescription dose was 14 Gy with a range of $12-16$ Gy to the $50 \%$ isodose line. In general, the dose would be reduced based on considerations related to tumor volume, proximity to critical structures such as the brainstem, preexisting neurological deficits, and history of previous treatments with radiosurgery. The total volume of brainstem receiving 10 and 12 Gy was restricted to less than $0.1 \mathrm{~cm}^{3}$ and $0.35 \mathrm{~cm}^{3}$, respectively. The dose to the optic nerve and chiasm was limited to a maximum dose of $8 \mathrm{~Gy}$. 
TABLE 1. Characteristics of 76 patients treated for CPA meningiomas

\begin{tabular}{|c|c|}
\hline Variable & Value \\
\hline No. of patients & 76 \\
\hline \multicolumn{2}{|l|}{$\operatorname{Sex}(\%)$} \\
\hline Male & $8(10.5)$ \\
\hline Female & $68(89.5)$ \\
\hline Median age in yrs (range) & $57(31-83)$ \\
\hline Median tumor size in cm (range) & $3.0(0.8-6.5)$ \\
\hline Surgery & $3.3(1.6-6.5)$ \\
\hline GKRS & $2.2(0.8-3.7)$ \\
\hline \multicolumn{2}{|l|}{ Initial therapeutic approach (\%) } \\
\hline Surgery & $51(67.1)$ \\
\hline GKRS & $25(32.9)$ \\
\hline \multicolumn{2}{|l|}{ WHO grade (\%) } \\
\hline 1 & $50(98.0)$ \\
\hline II & $1(2.0)$ \\
\hline Mean follow-up in mos (range) & $55(1-259)$ \\
\hline \multicolumn{2}{|l|}{ Presenting symptom (\%) } \\
\hline Hearing loss (CN VIII) & $26(34.2)$ \\
\hline Ataxia & $26(34.2)$ \\
\hline Facial numbness (CN V) & $19(25)$ \\
\hline Headaches & $14(18.4)$ \\
\hline Trigeminal neuralgia (CN V) & $14(18.4)$ \\
\hline Tinnitus (CN VIII) & $7(9.2)$ \\
\hline Vertigo (CN VIII) & $6(7.9)$ \\
\hline Diplopia (CN VI) & $4(5.3)$ \\
\hline Facial weakness (CN VII) & $3(3.9)$ \\
\hline Dysphagia (CNs IX, X, \& XII) & $2(2.6)$ \\
\hline Ptosis (CN III) & $1(1.3)$ \\
\hline
\end{tabular}

\section{Clinical and Neuroimaging Follow-Up}

Patients were monitored postoperatively using serial MRI within 6 to 12 months after the initial surgery to determine the size of any residual tumor and then every 6 to 12 months thereafter to evaluate for significant recurrence or progression until 2 years, at which point they were monitored less frequently. Significant growth was defined as tumor expansion of at least $5.0 \mathrm{~mm}$ in any direction on contrast-enhanced MRI compared with postoperative imaging, as previously described. ${ }^{9}$ Facial nerve function before and after intervention was documented according to the House-Brackmann facial nerve grading system. Good facial function was defined as House-Brackmann Grade I or II. ${ }^{24}$ The median follow-up for all patients included in this analysis was 46 months (range 2-259 months).

\section{Statistical Analysis}

Statistical analysis was performed using SPSS software (version 23.0, IBM) for Macintosh. Continuous variables are shown as the mean or median and range. Categorical values are given as percentages or frequencies and were analyzed where appropriate using the chi-square test. An independent-sample 2-tailed test was used to compare means. One-way ANOVA with unequal variance was used to compare means between multiple groups. A p value < 0.05 was considered statistically significant.

\section{Results}

\section{Patient and Tumor Characteristics}

We identified 76 patients during the past 24 years with meningiomas arising from the CPA that were initially treated with either GKRS or surgery performed by a single surgeon at our institution. Demographic information is provided in Table 1 . The median age was 57 years (range 31-83 years), and there was a clear sex predilection, with $68(89.5 \%)$ female patients and only $8(10.5 \%)$ male patients. The mean follow-up was 55 months (range 1-259 months). The median tumor size was $3.0 \mathrm{~cm}$ (range $0.8-6.5 \mathrm{~cm})$. Based on our treatment paradigm, $51(67.1 \%)$ patients underwent microsurgical resection, and 25 (32.9\%) patients underwent GKRS as primary treatment on initial diagnosis. The median tumor sizes were 3.25 $\mathrm{cm}$ (range 1.6-6.5 cm) and $2.2 \mathrm{~cm}$ (range $0.8-3.7 \mathrm{~cm}$ ) in patients treated initially with microsurgical resection and GKRS, respectively $(\mathrm{p}<0.001)$. Tissue diagnosis when available was consistent with WHO Grade I meningioma in $50(98.0 \%)$ patients and WHO Grade II meningioma in $1(2.0 \%)$ patient. A total of $36(47.4 \%)$ of the 76 patients ultimately received GKRS as a component of their therapy, and the one (1.3\%) patient with a WHO Grade II lesion underwent fractionated radiotherapy for disease progression. The most common presenting symptoms were hearing loss, ataxia, and facial numbness in $34 \%, 34 \%$, and $25 \%$ of patients, respectively. Additional presenting symptoms included both headaches and facial pain in $18.4 \%$ of patients, tinnitus, vertigo, diplopia, facial weakness, dysphagia, ptosis, and vision loss. Preoperative deficits were noted most frequently in CNs VIII (51.3\%) and V (43.4\%).

\section{Meningioma Origin}

Meningioma origin within the CPA and involvement of the IAC were identified on MR and/or CT imaging for all patients and corroborated with operative notes in surgically treated patients (Fig. 2 and Table 2). Meningioma attachment was most frequently classified as anterior petrous in $46(60.5 \%)$ patients, followed by posterior petrous in $19(25 \%)$ patients, petroclival in $6(7.9 \%)$ patients, and tentorial in $5(6.6 \%)$ patients. Tumor involved the IAC in $48(63.2 \%)$ patients. An arachnoid layer was present between the tumor and the contents of the IAC in $30(58.8 \%)$ patients who underwent surgical treatment, $16(31.4 \%)$ of whom had tumor involving the IAC. While tumor origin was not associated with IAC involvement $(p=0.703)$, tumor size larger than $3 \mathrm{~cm}$ was associated with involvement of the IAC $(p=0.011)$. Neither tumor origin nor tumor size was associated with the presence of an intact arachnoid boundary ( $\mathrm{p}=0.69$ and $\mathrm{p}=0.879$, respectively).

\section{Treatment Outcomes}

In patients who underwent aggressive microsurgical resection of their CPA meningioma by the facial nervesparing technique as described above, GTR was achieved in $34(66.7 \%)$ patients, and STR was achieved in 17 

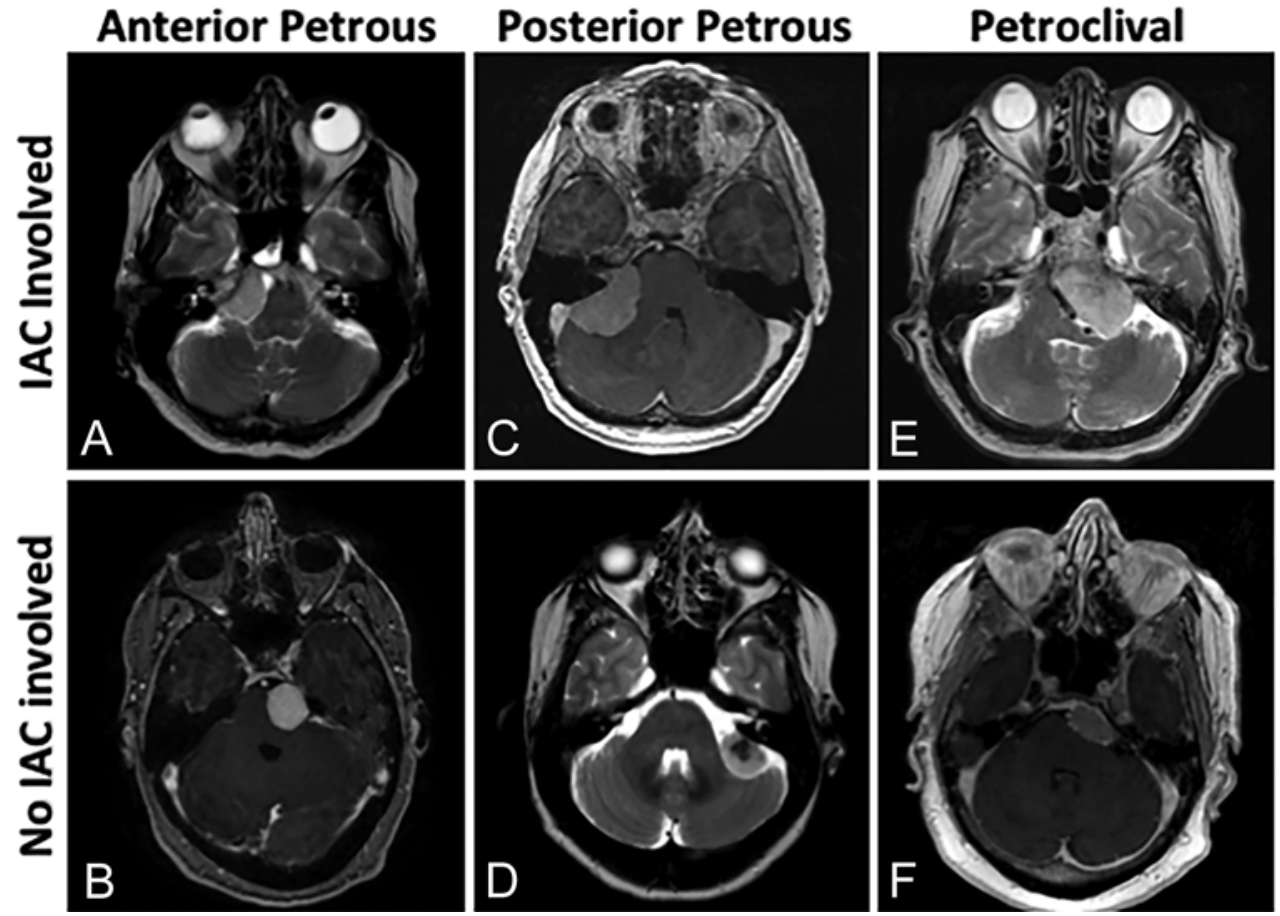
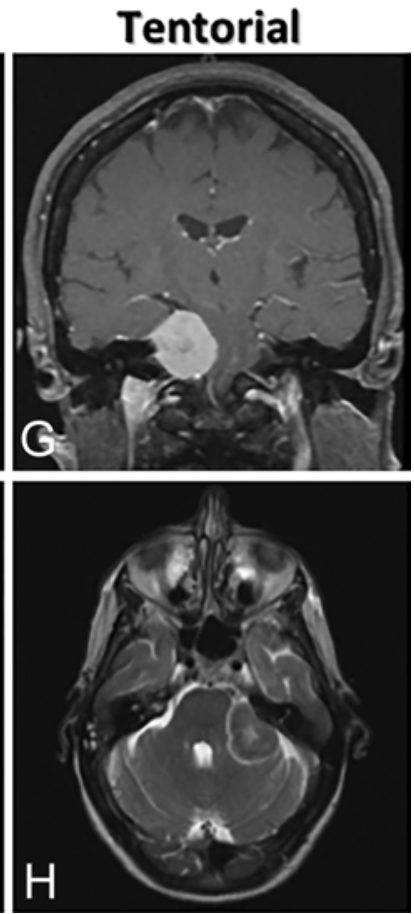

FIG. 2. Petrous origin-based classification of CPA meningiomas and IAC involvement as demonstrated on $T 2$-weighted (A, D, $\mathrm{E}$, and $\mathrm{H}$ ) or postcontrast T1-weighted (B, C, F, and G) MR images. Tumor origin was classified as petroclival if it arose from the petroclival junction medial to the trigeminal nerve displacing it laterally; anterior petrous if it arose along the petrous bone medial to the IAC and lateral to the trigeminal nerve, pushing it rostrally or medially; posterior petrous if it arose lateral to the IAC along the petrous bone; and tentorial if it arose from the tentorial edge and projected into the CPA, displacing the trigeminal nerve caudally. IAC involvement was documented. Tumor attachments and IAC involvement were confirmed intraoperatively.

(33.3\%) patients (Table 3). The median tumor size at the initial treatment was not significantly different between those receiving GTR $(3.33 \mathrm{~cm}$, range $2.0-6.5 \mathrm{~cm})$ or STR $(3.57 \mathrm{~cm}$, range $1.6-6.0 \mathrm{~cm} ; \mathrm{p}=0.925$ [Table 3]). Of note, 5 patients $(6.6 \%)$ received microsurgical resection despite tumor size smaller than $2.5 \mathrm{~cm}$ due to debilitating symptoms of vertigo or tinnitus, symptomatic mass effect on surrounding structures, or patient preference. Of these, 4 (5.3\%) patients underwent GTR and $1(1.3 \%)$ patient was treated with STR because of deep intracanalicular extension and the potential threat to CN VII. Similarly, tumor size was $2.5 \mathrm{~cm}$ or larger in $8(10.5 \%)$ patients who were initially treated with GKRS due to significant medical comorbidities, advanced age, or patient preference.

Tumor origin and the presence of IAC involvement were significantly associated with EOR. In surgically treated patients, the majority of tumors with anterior $(72.4 \%)$ and posterior $(73.3 \%)$ petrous origins underwent GTR $(p=$ 0.041, Table 2). All CPA meningiomas with a petroclival origin were treated with STR, typically in an effort to preserve CN VI. Evidence of IAC involvement resulted in GTR in $55.9 \%$ of surgically treated patients compared with $83.3 \%$ when there was no evidence of IAC involvement $(\mathrm{p}=0.048$, Table 2). The presence of an arachnoid boundary was not associated with EOR ( $p=0.07$, Table 2$)$.

Data on recurrence and progression are presented in Table 3. Meningiomas recurred or progressed in a total of $12(15.8 \%)$ patients. Of these, $5(14.7 \%)$ patients had previously undergone GTR and 7 (41.2\%) patients had
STR with known residual. EOR at initial treatment was not predictive of recurrence but showed a trend toward significance $(p=0.075)$. No patient treated initially with GKRS experienced disease progression over the course of follow-up. The median overall time to progression (TTP) in the surgical group was 3 years (range $0.2-9$ years), and the median TTP did not significantly differ based on GTR or STR at first surgery (2.5 vs 3 years, respectively; $p=$ $0.40)$. Tumor size at initial treatment was not associated with recurrence $(p=0.366)$.

Recurrence was treated using GKRS alone in a total of 7 (58.3\%) patients after a median TTP of 3.1 years (range $0.5-9$ years). Of these patients, 4 (57.1\%) had previously undergone GTR with a median TTP of 4.6 years (range $2.3-9$ years), and 3 (42.9\%) patients had previously undergone STR with a median TTP of 3.1 years (range 0.5-5.6 years; $p=0.131$ ). One patient with a known residual after STR of a petroclival meningioma underwent reoperation 4 years after initial resection and ultimately required adjuvant GKRS for progression at 8 months and again at 12 years after a second STR. Another patient with a known residual after STR for an anterior petrous meningioma underwent reoperation 3 years after the initial resection and ultimately required adjuvant GKRS for progression at 1 month and again at 1 year after the second STR. A third patient with known residual anterior petrous meningioma underwent GKRS for recurrent symptoms at 2 months and received a second round of GKRS 5 years after initial resection before ultimately undergoing repeat resection 6 
TABLE 2. Tumor origin and EOR

\begin{tabular}{|c|c|c|c|c|c|}
\hline \multirow[b]{2}{*}{ Variable } & \multirow[b]{2}{*}{ Total (\%) } & \multirow{2}{*}{$\begin{array}{l}\text { Median Tumor Size in } \\
\mathrm{cm} \text { (range) }\end{array}$} & \multicolumn{3}{|c|}{ Therapeutic Approach (\%) } \\
\hline & & & GTR & STR & GKRS \\
\hline \multicolumn{6}{|l|}{ Origin* } \\
\hline Anterior petrous & $46(60.5)$ & $2.8(1.0-6.5)$ & $21(72.4)$ & $8(27.6)$ & $17(37.0)$ \\
\hline IAC & $29(63.0)$ & $3.0(1.8-6.5)$ & $12(63.2)$ & $7(36.8)$ & $10(13.2)$ \\
\hline No IAC & $17(37.0)$ & $2.5(1.0-5.5)$ & $9(81.8)$ & $2(18.2)$ & $6(7.9)$ \\
\hline Posterior petrous & $19(25)$ & $3.6(0.8-6.0)$ & $11(73.3)$ & $4(26.7)$ & $4(5.3)$ \\
\hline IAC & $13(68.4)$ & $3.6(0.8-6.0)$ & $6(60.0)$ & $4(40.0)$ & $3(3.9)$ \\
\hline No IAC & $6(46.2)$ & $3.5(0.8-4.0)$ & $5(100.0)$ & $0(0)$ & $1(1.3)$ \\
\hline Petroclival & $6(7.9)$ & $3.0(1.8-4.5)$ & $0(0)$ & $4(100.0)$ & $2(2.6)$ \\
\hline IAC & $4(66.7)$ & $3.4(2.2-4.5)$ & $0(0)$ & $3(100.0)$ & $1(1.3)$ \\
\hline No IAC & $2(33.3)$ & $2.2(1.8-2.6)$ & $0(0)$ & $1(100.0)$ & $1(1.3)$ \\
\hline Tentorial & $5(6.6)$ & $2.5(1.5-4.0)$ & $2(66.7)$ & $1(33.3)$ & $2(2.6)$ \\
\hline IAC & $2(40.0)$ & $3.5(3.0-4.0)$ & $1(50.0)$ & $1(50.0)$ & $0(0)$ \\
\hline No IAC & $3(60.0)$ & $2.1(1.5-2.5)$ & $1(100.0)$ & $0(0)$ & $2(2.6)$ \\
\hline \multicolumn{6}{|l|}{ IAC involvement $†$} \\
\hline Yes & $48(63.2)$ & $3.0(3.0-6.5)$ & $19(55.9)$ & $15(44.1)$ & $14(18.4)$ \\
\hline No & $28(36.8)$ & $2.5(1.5-5.5)$ & $15(83.3)$ & $3(16.7)$ & $10(13.2)$ \\
\hline \multicolumn{6}{|c|}{ Intact arachnoid boundary $\ddagger$} \\
\hline Yes & $30(39.5)$ & $3.3(2.0-6.5)$ & $23(76.7)$ & $7(23.3)$ & NA \\
\hline No & $21(27.6)$ & $3.4(1.6-6.0)$ & $11(52.4)$ & $10(47.6)$ & NA \\
\hline $\begin{array}{l}\text { NA = not applicable. } \\
\text { * Tumor origin was associa } \\
\dagger \text { Tumor origin was not ass } \\
\ddagger \text { Tumor origin was not ass } \\
\text { not associated with EOR (p }\end{array}$ & $\begin{array}{l}=0.041) \text {. } \\
\text { involvemen } \\
\text { presence of }\end{array}$ & $\begin{array}{l}=0.703) ; \text { IAC involvement } \\
\text { ntact arachnoid boundary }\end{array}$ & $\begin{array}{l}\text { ssociated wit } \\
.69) ; \text { the pres }\end{array}$ & $\begin{array}{l}R(p=0.048 \\
\text { of an arachr }\end{array}$ & undary was \\
\hline
\end{tabular}

years after initial resection. Recurrence was not associated with tumor origin at initial diagnosis, IAC involvement, or the presence of an arachnoid plane $(\mathrm{p}=0.543, \mathrm{p}=0.740$, and $\mathrm{p}=1.000$, respectively).

\section{Facial Nerve Preservation}

In an effort to characterize preservation of facial nerve motor function using our treatment algorithm, we examined pre- and postoperative facial nerve function in all patients treated with either microsurgical resection or GKRS (Table 4 and Fig. 3). On initial presentation, facial nerve motor weakness was classified as House-Brackmann Grade III or worse in 3 (3.9\%) patients, all of whom under- went a facial nerve-sparing microsurgical resection. Posttreatment (surgery or GKRS), excellent facial nerve motor function, defined as House-Brackmann Grade I or II, was present in $68(89.5 \%)$ patients. Facial nerve function was unchanged from initial evaluation in $66(86.8 \%)$ patients, $41(53.9 \%)$ of whom had been treated with resection, and improved in $2(2.6 \%)$ patients, both of whom underwent resection. Postoperatively, facial nerve function was classified as House-Brackmann Grade I or II in 43 (84.3\%) patients, while facial nerve motor function and HouseBrackmann grades remained unchanged in all patients $(100 \%)$ who received GKRS as initial therapy.

Facial nerve motor function improved in 2 of the 3

TABLE 3. Recurrence/progression characteristics in patients treated with surgery and/or GKRS

\begin{tabular}{|c|c|c|c|c|}
\hline \multirow[b]{2}{*}{ Characteristic } & \multirow[b]{2}{*}{ Total } & \multicolumn{3}{|c|}{ Initial Therapeutic Approach } \\
\hline & & GTR & STR & GKRS \\
\hline No. of patients treated (\%) & 76 & $34(66.7)$ & $17(33.3)$ & $25(32.9)$ \\
\hline Median tumor size in $\mathrm{cm}$ (range) ${ }^{*}$ & $3.0(0.8-6.5)$ & $3.33(2.0-6.5)$ & $3.57(1.6-6.0)$ & $2.13(0.8-3.7)$ \\
\hline Recurrences $(\%) \dagger$ & $12(15.8)$ & $5(14.7)$ & $7(41.2)$ & $0(0)$ \\
\hline Median time to recurrence in yrs (range) $\ddagger$ & $3(0.2-9)$ & $2.5(1.3-9)$ & $3.0(0.2-5.6)$ & NA \\
\hline
\end{tabular}


TABLE 4. Facial nerve outcomes

\begin{tabular}{|c|c|c|c|}
\hline \multirow[b]{2}{*}{ Variable } & \multicolumn{3}{|c|}{ Facial Nerve Outcome (\%) } \\
\hline & Unchanged & Improved & Worsened \\
\hline Total & $66(86.8)$ & $2(2.6)$ & $8(10.5)$ \\
\hline \multicolumn{4}{|l|}{ Therapeutic approach } \\
\hline Surgery & $41(80.4)$ & $2(3.9)$ & $8(15.7)$ \\
\hline GKRS & $25(32.9)$ & $0(0)$ & $0(0)$ \\
\hline $\begin{array}{l}\text { Median tumor size, } \mathrm{cm} \\
(\text { range })^{*}\end{array}$ & $2.9(0.8-6.5)$ & 4.0 & $4.0(2.5-6)$ \\
\hline \multicolumn{4}{|l|}{ Arachnoid plane $†$} \\
\hline Yes & $29(96.7)$ & $0(0)$ & $1(3.3)$ \\
\hline No & $12(57.1)$ & $2(9.5)$ & $7(33.3)$ \\
\hline \multicolumn{4}{|l|}{ IAC involvement $\ddagger$} \\
\hline Yes & $40(83.3)$ & $2(4.2)$ & $6(12.5)$ \\
\hline No & $26(92.9)$ & $0(0)$ & $2(7.1)$ \\
\hline \multicolumn{4}{|l|}{ Origin } \\
\hline Anterior petrous & $41(89.1)$ & $1(2.2)$ & $4(8.7)$ \\
\hline Anterior petrous $+\mathrm{IAC}$ & $25(86.2)$ & $1(3.4)$ & $3(10.3)$ \\
\hline Posterior petrous & $17(89.5)$ & $0(0)$ & $2(10.5)$ \\
\hline Posterior petrous + IAC & $12(92.3)$ & $0(0)$ & $1(7.7)$ \\
\hline Petroclival & $4(66.7)$ & $0(0)$ & $2(33.3)$ \\
\hline Petroclival + IAC & $2(50.0)$ & $0(0)$ & $2(50.0)$ \\
\hline Tentorial & $4(80.0)$ & $1(20.0)$ & $0(0)$ \\
\hline Tentorial + IAC & $1(50.0)$ & $1(50.0)$ & $0(0)$ \\
\hline \multicolumn{4}{|l|}{ EOR } \\
\hline GTR & $30(88.2)$ & $2(5.9)$ & $2(5.9)$ \\
\hline STRß & $12(66.7)$ & $0(0)$ & $6(33.3)$ \\
\hline
\end{tabular}

Values in parentheses are percentages except where noted otherwise.

* Large tumor size was associated with worsened facial nerve function postoperatively $(p=0.017)$.

$\dagger$ The presence of an arachnoid plane was associated with better facial nerve outcomes $(p=0.002)$.

$\ddagger I A C$ involvement was not associated with worsened facial nerve function postoperatively $(p=0.400)$.

$\S$ STR was associated with permanent postoperative facial nerve dysfunction.

patients who presented initially with facial nerve motor weakness and worsened transiently in only 1 patient, who was ultimately left with mild permanent weakness classified as House-Brackmann Grade II. Of the 2 patients with improved facial nerve motor function, 1 patient improved from House-Brackmann Grade IV to Grade III after resection and the other improved from House-Brackmann Grade III to Grade II. Overall, facial nerve function worsened in $8(10.5 \%)$ patients, and permanent facial nerve motor deficits-defined as greater than House-Brackmann Grade III-were identified in a total of $8(10.5 \%)$ patients in this series.

Larger tumor size was associated with worsened facial nerve function postoperatively. The median tumor size in patients with unchanged postoperative facial nerve motor function was $2.9 \mathrm{~cm}$ (range $0.8-6.5 \mathrm{~cm}$ ) compared with $4.0 \mathrm{~cm}$ (range $2.5-6 \mathrm{~cm}$ ) in patients with worsened facial nerve motor function $(\mathrm{p}=0.017)$. Tumor origin was not associated with permanent facial nerve dysfunction $(p=0.108)$. Interestingly, IAC involvement was not significantly associated with worsened facial nerve function $(p=0.400)$, despite IAC involvement in $75 \%$ of patients with worsened facial nerve outcomes. Facial nerve function was unchanged or improved in $32(94 \%)$ patients who underwent GTR of their lesion and worsened in $2(5.9 \%)$ patients. In contrast, facial nerve function was unchanged or improved in $12(66.7 \%)$ patients who underwent STR and worsened in $6(33.3 \%)$ patients (Table 4). Thus, permanent postoperative facial nerve dysfunction occurred more frequently in patients who underwent STR $(p=0.024)$.

Intraoperative neuromonitoring changes were only encountered in $2(3.9 \%)$ patients. In one patient, facial nerve EMG was noted to be diminished proximally, requiring higher stimulations greater than $0.1 \mathrm{~mA}$ above baseline along the course of the facial nerve with preserved distal stimulation. In the other patient, at the end of the case, only partial depolarizations of the facial nerve were noted with stimulations greater than $0.3 \mathrm{~mA}$. Both patients developed postoperative facial nerve weakness $(p=0.002)$.

Presence of an arachnoid boundary was encountered in $30(58.8 \%)$ patients treated surgically, in whom facial nerve function was unchanged or improved in 29 (96.7\%). Absence of an arachnoid boundary between the tumor and CN VII was encountered in $21(41.2 \%)$ patients, of which $18(85.7 \%)$ involved the IAC. Seven (33.3\%) patients without intraoperative evidence of an intact arachnoid plane separating tumor and CN VII had worsened postoperative facial motor nerve function, with IAC involvement noted in $5(71.4 \%)$ of these patients. The presence of an arachnoid plane at initial resection predicted better facial nerve outcomes, as $96.7 \%$ of patients with an intact arachnoid boundary had unchanged or improved facial nerve function compared with $57 \%$ in patients without an intact boundary $(\mathrm{p}=0.002)$.

\section{Complications}

Postoperative complications are reported in Table 5. Including CN VII dysfunction, new and permanent postoperative cranial neuropathies were noted in 12 (23.5\%) patients and included CN VI (7.8\%) and CN X (5.8\%). Four $(7.8 \%)$ patients suffered CSF leaks. Hydrocephalus requiring CSF diversion via ventriculoperitoneal shunt insertion occurred in $3(5.8 \%)$ patients and lumboperitoneal shunt in $1(2.0 \%)$ patient. Tumor size was not significantly associated with hydrocephalus $(\mathrm{p}=0.977)$. There were no perioperative deaths. One patient required emergency reoperation for suboccipital decompression following a cerebellar infarction and made a complete recovery. No patient treated initially with GKRS developed hydrocephalus or required ventriculoperitoneal shunt insertion. Interestingly, 1 patient treated with GKRS did develop a superior cerebellar artery aneurysm 10 years posttreatment, which was subsequently treated with coiling and vessel sacrifice. ${ }^{11}$

\section{Discussion}

Meningiomas, second in frequency only to vestibular schwannomas, account for 5\% to $15 \%$ of tumors arising within the CPA and typically present with headaches and lower cranial nerve findings. ${ }^{6,20}$ While aggressive and complete resection of meningiomas is curative, tumors arising within the CPA present unique surgical challenges com- 

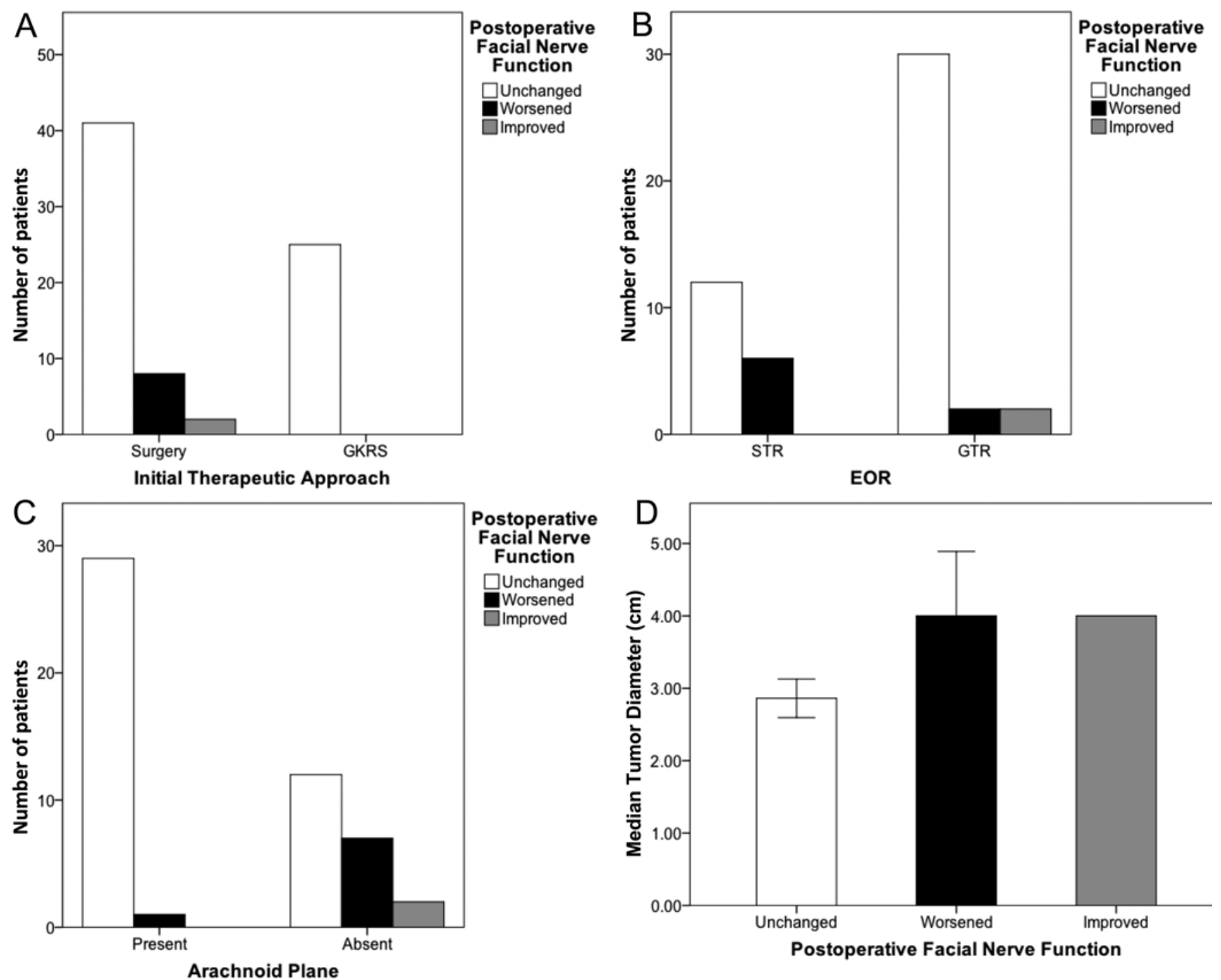

FIG. 3. Predictors of postoperative facial nerve motor function. Facial nerve motor function was unchanged in 41 patients and improved in 2 patients treated initially with surgery, and was preserved in 25 patients initially treated with GKRS. A: Facial motor nerve function worsened in 8 patients, all of whom were treated with resection. B: Permanent postoperative facial nerve dysfunction was associated with STR $(p=0.024)$. C: An arachnoid plane was associated with facial nerve outcomes, as $96.7 \%$ of patients with an intact arachnoid boundary had unchanged or improved facial nerve function compared with $57 \%$ in patients without an intact boundary $(p=0.002)$. D: Tumor size was associated with worsened facial nerve function postoperatively. The median tumor size in patients with unchanged postoperative facial nerve motor function was $2.9 \mathrm{~cm}$ (range $0.8-6.5 \mathrm{~cm}$ ) compared with $4.0 \mathrm{~cm}$ (range 2.5-6 cm) in patients with worsened facial nerve motor function $(p=0.017)$.

pared with supratentorial lesions, given their close association with critical neurological and vascular structures., ${ }^{1,5,21}$ As a result, GTR cannot always be accomplished without placing the patient at significant risk of morbidity and mortality. In an effort to preserve neurological function, a more conservative surgical approach has evolved for the management of CPA meningiomas. When GTR is deemed unsafe, STR guided by the relief of mass effect and the preservation of neurological function, with the option for repeat surgery or GKRS for progression of residual tumor, is now an acceptable treatment paradigm.,25

Over the past 24 years, we have used a treatment algorithm for tumors of the CPA in which we use GKRS for smaller tumors $(<2.5 \mathrm{~cm})$ without associated mass effect or progressive neurological symptoms, and we reserve conservative resection for larger lesions with special attention given to preserving neurological function regardless of EOR. We have previously published on the safety and efficacy of this paradigm for the resection of vestibular schwannomas where facial nerve motor function (defined as House-Brackmann Grade I or II) was preserved in $97 \%$ of patients. ${ }^{9}$ However, the safety and efficacy of this approach in CPA meningiomas has not been well examined. In the present series, we review our experience and outcomes with treating meningiomas of the CPA in one of the largest series by a single surgeon to date. Specifically, we examine preservation of facial nerve function and investigate the anatomy of the tumor relative to the IAC as a possible prognosticator for postoperative facial nerve dysfunction. We hypothesize that this treatment paradigm results 
TABLE 5. Surgical complications

\begin{tabular}{lc}
\hline \multicolumn{1}{c}{ Complication } & No. of Patients (\%) \\
\hline Hydrocephalus + shunt & $3(5.9)$ \\
\hline CSF leak & $4(7.8)$ \\
\hline Infection & $2(3.9)$ \\
\hline New cranial neuropathy & $12(23.5)$ \\
\hline Vascular complications & $1(2.0)$ \\
\hline Cerebellar infarct & $1(2.0)$ \\
\hline
\end{tabular}

in acceptable clinical outcomes with minimal associated risk. We also examine rates of recurrence and complications associated with our treatment paradigm.

Resection remains the mainstay of treatment for CPA meningiomas, with variable rates of resection and associated morbidity reported. In a recent retrospective review of 34 patients who underwent surgery for CPA meningiomas, $19(55.9 \%)$ patients received GTR and $15(44.1 \%)$ patients underwent STR; there was a significant decrease in the rate of GTR to $9.1 \%$ in patients with tumors larger than 3 $\mathrm{cm}$ because of the complexity of these lesions. ${ }^{1}$ Leonetti et al. were able to achieve GTR in $19(66 \%)$ of 29 patients with CPA meningiomas larger than $3 \mathrm{~cm}$ using a combined retrosigmoid-transpetrosal-transcochlear approach to increase exposure and thus mitigate the threat to critical neurovascular structures at the cost of hearing preservation. ${ }^{15}$ In the present series, 51 patients underwent resection for CPA meningiomas, with $34(67 \%)$ patients receiving GTR and $17(33 \%)$ patients receiving STR using exclusively a retrosigmoid approach in large lesions $(\geq 2.5 \mathrm{~cm})$.

Interestingly, tumor origin was associated with EOR in this series. Using a classification system developed by $\mathrm{Ku}-$ nii et al., we classified treated CPA meningiomas as arising from the tentorium, the petroclival region, the anterior petrous bone, or the posterior petrous bone. ${ }^{13}$ Involvement of the IAC was noted to occur in $40(52.6 \%)$ patients, similar to that reported in the literature. , $^{1710,13,19}$ Tumors arising from the anterior and posterior petrous dura were more likely to undergo GTR than tumors arising from the petroclival region or the tentorium $(\mathrm{p}=0.041)$. Petroclival tumors uniformly underwent STR, likely reflecting their deep anterior attachment and the surgeon's efforts to preserve CN VI as it traversed the operative field. Regardless of origin, meningiomas with IAC involvement were more likely to receive STR ( $p=0.048)$, confirming the surgeon's efforts to preserve facial nerve function even in the setting of leaving residual tumor. Interestingly, while tumor size greater than $3 \mathrm{~cm}$ was associated with involvement of the IAC ( $\mathrm{p}=0.011)$, it was not associated with EOR ( $\mathrm{p}=$ $0.925)$, suggesting that the anatomical relationship of the tumor and its involvement of critical neurological and vascular structures may better define its ability to be resected completely and safely.

In the present series, rates of progression or recurrence were low and unrelated to EOR, tumor origin, IAC involvement, or the presence of an arachnoid plane between the tumor and critical structures. A total of 7 (13.7\%) patients treated initially surgically ultimately received GKRS for progression or recurrence, while $5(9.8 \%)$ patients had more complicated long-term management involving reop- eration and repeat GKRS. This low rate of progression is in contrast to a recent large, multicenter investigation of outcomes after GKRS for posterior fossa meningiomas, reporting that $43.3 \%$ of patients had undergone prior surgical management. However, that study included meningiomas arising within the posterior fossa as a whole and did not comment on EOR prior to radiosurgery. Importantly, the authors in that study reported tumor control rates of $95 \%, 92 \%$, and $81 \%$ at 3,5 , and 10 years after radiosurgery, respectively. ${ }^{20,22}$ Similarly, we found that GKRS as the initial treatment for small, mildly symptomatic lesions as well as recurrent or progressive tumors resulted in excellent disease control, and no patient treated initially with GKRS experienced progression over the follow-up period. Low rates of progression/recurrence suggest that longterm disease control for these low-grade lesions is possible with the outlined treatment algorithm, even in the setting of STR, and supports the goal of preserving neurological function whenever possible to maximize patient quality of life. While requiring $5.0 \mathrm{~mm}$ of growth in any direction can be considered restrictive for diagnosing progression or recurrence, our anecdotal experience shows that this value eliminates ambiguity due to differences in imaging techniques and interobserver variation among different radiologists. Importantly, while tumor recurrence or progression did not significantly vary based on initial EOR, a lower rate of recurrence or progression after GTR approached significance $(\mathrm{p}=0.075)$. While this may be the result of the small number of recurrences, it is possibly due to residual microscopic disease within the dura despite radiographic evidence of GTR. As a result, monitoring of patients who underwent GTR is recommended.

Morbidity, and, in particular, rates of cranial nerve dysfunction, with resection for CPA meningiomas, ranges from $10.4 \%$ to $35.7 \%$ and tends to increase with tumor size. ${ }^{1,5,15}$ Facial nerve (CN VII) palsy is a particularly adverse outcome of surgery within the CPA, occurring in 6\% to $29 \%$ of patients and causing disfiguring and disabling facial paralysis that detracts from a patient's quality of

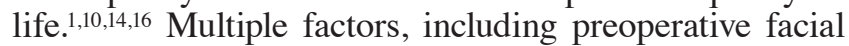
nerve status, surgical approach, anatomical location, and involvement of the IAC, likely contribute to perioperative CN VII dysfunction. ${ }^{1,10,17,18,23}$ In the study by Agarwal et al., permanent $\mathrm{CN}$ complications were reported in 5.9\% patients with tumors smaller than $3 \mathrm{~cm}$ and in $45.5 \%$ of the cases in which the lesions were larger than $3 \mathrm{~cm}$ and were more frequent in those undergoing STR than in those undergoing GTR. ${ }^{1}$ Similarly, Leonetti et al. reported permanent postoperative complications in $8(27.6 \%)$ patients, with CN VII dysfunction specifically being reported in 3 $(10.3 \%)$ patients..$^{15}$ In the present series, facial nerve motor function was unchanged or improved in 68 (89.5\%) patients and worsened in $8(10.5 \%)$ patients, all of whom had undergone initial surgical management, and of whom 1 patient already had preoperative facial weakness. Similarly, we noted that both increased tumor size and STR were associated with permanent facial nerve motor deficit $(p=0.017$ and $p=0.024$, respectively). This again likely reflects the complexity of surgical intervention for large CPA meningiomas, as they have a broader interface with the brainstem and/or traversing cranial nerves, leading to 
the surgeon's hesitancy to risk neurological damage. New cranial neuropathies were otherwise encountered in $23.5 \%$ of patients treated surgically and most frequently involved CNs VI and X.

We wanted to investigate whether specific anatomical features of CPA meningiomas and their location relative to the IAC played a role in the threat of permanent facial nerve dysfunction following resection. In particular, as tumors grow from their point of attachment, normal anatomy may be displaced or completely engulfed within tumor, complicating the identification and, ultimately, preservation of normal anatomy during resection. The presence or absence of an intact arachnoid boundary between the tumor and the contents of the IAC may suggest the risk of cranial nerve damage. In the present series, tumor origin and IAC involvement were not significantly associated with postoperative facial weakness $(\mathrm{p}=0.108$ and $\mathrm{p}$ $=0.400$, respectively), although $75 \%$ of patients with facial nerve dysfunction had evidence of IAC involvement. Interestingly, the presence of an arachnoid plane between the tumor and the IAC contents was protective of CN VII function even in cases in which pieces of tumor extended into the canal $(\mathrm{p}=0.002)$.

In the present series, no patient treated with GKRS developed facial nerve dysfunction. Interestingly, evidence suggests that increasing tumor volume and CPA location are associated with neurological decline after radiosurgery alone and likely reflects the narrow corridor of the CPA and its intolerance of edema, resulting in compression of critical structures. ${ }^{20}$ As a result, in the setting of larger CPA tumors, the benefits seen with smaller tumor volumes at the time of radiosurgery highlight the importance of an initial cytoreductive surgery. We suggest that GTR be attempted with a low threshold to pursue STR with adjuvant GKRS for radiographic or symptom progression.

\section{Limitations}

While the present report is one of the largest surgical series to date focused on CPA meningiomas, there are some limitations of this retrospective analysis that are worth noting. The low patient numbers in each cohort limits post hoc statistical analysis based on anatomical origin. Inherent selection biases and a lack of a control arm seen in retrospective studies are other notable limitations. Also, not all tumors were histologically confirmed to be meningioma. Thus, however unlikely, it is possible that other benign skull base lesions were included in this study.

\section{Conclusions}

The CPA is a narrow corridor traversed by critical neurovascular structures. In the setting of large CPA tumors, identification of CN VII is paramount to preservation of facial motor nerve function. As tumors enlarge, normal anatomy may be displaced or completely engulfed within tumor, complicating the identification and ultimately preservation of normal anatomy during resection. A viable consideration should include STR of the portion of the tumor located within and near the CPA with adjuvant GKRS in the setting of radiographic or symptomatic progression of residual tumor. This paradigm results in acceptable rates of GTR, when possible, and excellent preservation of facial nerve motor function.

\section{References}

1. Agarwal V, Babu R, Grier J, Adogwa O, Back A, Friedman $\mathrm{AH}$, et al: Cerebellopontine angle meningiomas: postoperative outcomes in a modern cohort. Neurosurg Focus 35(6):E10, 2013

2. Asthagiri AR, Helm GA, Sheehan JP: Current concepts in management of meningiomas and schwannomas. Neurol Clin 25:1209-1230, xi, 2007

3. Barbaro NM, Gutin PH, Wilson CB, Sheline GE, Boldrey EB, Wara WM: Radiation therapy in the treatment of partially resected meningiomas. Neurosurgery 20:525-528, 1987

4. Black PM, Villavicencio AT, Rhouddou C, Loeffler JS: Aggressive surgery and focal radiation in the management of meningiomas of the skull base: preservation of function with maintenance of local control. Acta Neurochir (Wien) 143:555-562, 2001

5. Chen CM, Huang AP, Kuo LT, Tu YK: Contemporary surgical outcome for skull base meningiomas. Neurosurg Rev 34:281-296, 2011

6. Friedmann DR, Grobelny B, Golfinos JG, Roland JT Jr: Nonschwannoma tumors of the cerebellopontine angle. Otolaryngol Clin North Am 48:461-475, 2015

7. Gao K, Ma H, Cui Y, Chen X, Ma J, Dai J: Meningiomas of the cerebellopontine angle: radiological differences in tumors with internal auditory canal involvement and their influence on surgical outcome. PLoS One 10:e0122949, 2015

8. Goldsmith BJ, Wara WM, Wilson CB, Larson DA: Postoperative irradiation for subtotally resected meningiomas. A retrospective analysis of 140 patients treated from 1967 to 1990. J Neurosurg 80:195-201, 1994

9. Haque R, Wojtasiewicz TJ, Gigante PR, Attiah MA, Huang B, Isaacson SR, et al: Efficacy of facial nerve-sparing approach in patients with vestibular schwannomas. J Neurosurg 115:917-923, 2011

10. Kane AJ, Sughrue ME, Rutkowski MJ, Berger MS, McDermott MW, Parsa AT: Clinical and surgical considerations for cerebellopontine angle meningiomas. J Clin Neurosci 18:755-759, 2011

11. Kellner CP, McDowell MM, Connolly ES Jr, Sisti MB, Lavine SD: Late onset aneurysm development following radiosurgical obliteration of a cerebellopontine angle meningioma. J Neurointerv Surg 7:e21, 2015

12. Kondziolka D, Mathieu D, Lunsford LD, Martin JJ, Madhok R, Niranjan A, et al: Radiosurgery as definitive management of intracranial meningiomas. Neurosurgery 62:53-60, 2008

13. Kunii N, Ota T, Kin T, Kamada K, Morita A, Kawahara N, et al: Angiographic classification of tumor attachment of meningiomas at the cerebellopontine angle. World Neurosurg 75:114-121, 2011

14. Lassaletta L, Alfonso C, Del Rio L, Roda JM, Gavilan J: Impact of facial dysfunction on quality of life after vestibular schwannoma surgery. Ann Otol Rhinol Laryngol 115:694698,2006

15. Leonetti JP, Anderson DE, Marzo SJ, Origitano TC, Schuman R: Combined transtemporal access for large ( $>3$ $\mathrm{cm})$ meningiomas of the cerebellopontine angle. Otolaryngol Head Neck Surg 134:949-952, 2006

16. Martin HC, Sethi J, Lang D, Neil-Dwyer G, Lutman ME, Yardley L: Patient-assessed outcomes after excision of acoustic neuroma: postoperative symptoms and quality of life. J Neurosurg 94:211-216, 2001

17. Nakamura M, Roser F, Dormiani M, Matthies C, Vorkapic P, Samii M: Facial and cochlear nerve function after surgery of cerebellopontine angle meningiomas. Neurosurgery 57:7790,2005 
18. Nakamura M, Roser F, Mirzai S, Matthies C, Vorkapic P, Samii M: Meningiomas of the internal auditory canal. Neurosurgery 55:119-128, 2004

19. Park SH, Kano H, Niranjan A, Flickinger JC, Lunsford LD: Stereotactic radiosurgery for cerebellopontine angle meningiomas. J Neurosurg 120:708-715, 2014

20. Sheehan JP, Starke RM, Kano H, Barnett GH, Mathieu D, Chiang V, et al: Gamma Knife radiosurgery for posterior fossa meningiomas: a multicenter study. J Neurosurg 122:1479-1489, 2015

21. Simpson D: The recurrence of intracranial meningiomas after surgical treatment. J Neurol Neurosurg Psychiatry 20:22-39, 1957

22. Starke RM, Nguyen JH, Rainey J, Williams BJ, Sherman JH, Savage J, et al: Gamma Knife surgery of meningiomas located in the posterior fossa: factors predictive of outcome and remission. J Neurosurg 114:1399-1409, 2011

23. von Eckardstein KL, Driscoll CL, Link MJ: Outcome after microsurgery for meningiomas involving the internal auditory canal. Neurosurgery 67:1236-1242, 2010

24. Vrabec JT, Backous DD, Djalilian HR, Gidley PW, Leonetti JP, Marzo SJ, et al: Facial Nerve Grading System 2.0. Otolaryngol Head Neck Surg 140:445-450, 2009

25. Zachenhofer I, Wolfsberger S, Aichholzer M, Bertalanffy A, Roessler K, Kitz K, et al: Gamma-Knife radiosurgery for cranial base meningiomas: experience of tumor control, clini- cal course, and morbidity in a follow-up of more than 8 years. Neurosurgery 58:28-36, 2006

\section{Disclosures}

The authors report no conflict of interest concerning the materials or methods used in this study or the findings specified in this paper.

\section{Author Contributions}

Conception and design: D'Amico, Malone, Sisti. Acquisition of data: D’Amico, Banu, Petridis, Bercow, Praver. Analysis and interpretation of data: D'Amico, Banu, Petridis. Drafting the article: D'Amico. Critically revising the article: D'Amico, Banu, Malone, Praver, Wang, Sisti. Reviewed submitted version of manuscript: D’Amico, Malone, Wang, Sisti. Statistical analysis: Banu. Administrative/technical/material support: Isaacson, Sisti. Study supervision: D’Amico, Sisti.

\section{Correspondence}

Randy S. D'Amico, Columbia University Medical Center, Neurological Surgery, 710 W 168th St., New York, NY 10032. email: rd2398@cumc.columbia.edu. 